\title{
Pharmaceutical Quality/CMC Interpretation Code (text) Terminology
}

National Cancer Institute

\section{Source}

National Cancer Institute. Pharmaceutical Quality/CMC Interpretation Code (text)

Terminology. NCl Thesaurus. Code C133857.

A category of terminology used to qualify the information pertaining to text interpretation codes in the framework of the Pharmaceutical Quality/Chemistry, Manufacturing and Controls documents. 